\title{
La doctrine dans les hymnes de la liturgie wisigothique
}

\section{Entre tradition patristique et réécriture biblique}

La liturgie hispanique, qui conserva sa spécificité jusqu'à sa suppression par Rome en 1089, comprend un corpus d'hymnes propres particulièrement important - 210 dans l'édition de référence de J. Castro Sánchez ${ }^{1}$. Il s'agit de pièces de mètre et de mesure variés, pour la plupart rythmiques et recherchant la rime ${ }^{2}$. Ces hymnes relevant de la liturgie des heures sont divisés, depuis $\mathrm{Cl}$. Blume ${ }^{3}$, en cinq groupes d'importance inégale : 1 . Propre du temps (1-43); Commun du temps (44-80); Propre des Saints (81-179) ; Commun des Saints (180-186) ; Occasions diverses (187-210). On connaît le nom de plusieurs hymnographes dont Maxime et Jean, évêques de Saragosse, Isidore de Séville, Eugène de Tolède ou encore Quiricus de Barcelone aux $\mathrm{VI}^{\mathrm{e}}$ et $\mathrm{VII}^{\mathrm{e}}$ siècles. Cependant, presque tous ces poèmes nous sont parvenus de manière anonyme, dans des manuscrits très tardifs où ils sont mêlés à des hymnes empruntés à d'autres liturgies, d'où les sérieuses difficultés que pose parfois leur datation.

Le corpus est évidemment trop vaste et trop hétérogène pour être considéré dans son ensemble ici. Notre attention ira, en accord avec nos thèmes de recherche actuels et la grande fortune que connut ce genre à l'époque wisigothique, aux quelque cinquante hymnes qui furent, de manière certaine ou probable, composés entre la conversion des Wisigoths (589) et l'invasion arabe $(711)^{4}$. Au cours de cette période, la création liturgique fut en effet particulièrement florissante en Hispanie, même si la

1 Castro Sánchez (2010), qui remplace désormais Blume (1897). Tous les textes seront cités d'après cette édition, et accompagnés d'une traduction personnelle. De fait, ces hymnes n'ont été traduits qu'en castellan par Arana Tarazona (2016) d'après l'édition de Blume, et en espagnol par Castro Sánchez / García Ruiz (2014) d'après l'édition de Castro Sánchez (avec des annotations sommaires). La traduction française donnée par Guéranger (1841) de l'hymne Gaudete flores martirum est très libre. - Parmi les contributions récentes sur l'hymnodie hispanique en général prenant en considération les hymnes de la période wisigothique, on renverra, outre les références précitées, à Szövérffy (1998) ; Valcárcel Martínez (2005) ; Arocena-Solano (2012) ; sur les hymnes du temporal antérieurs à l'invasion musulmane, De Almeida Matos (1977).

2 Cf. Almeida Matos (1984) qui se fonde, entre autres, sur l'hymne Auctor luminis étudié infra. - Les dates de composition avancées par l'auteur sur la base de ses analyses métriques sont désormais dépassées.

3 Blume (1897). Cette typologie est commentée par Castro Sánchez / García Ruiz (2014) 17-19.

4 À l'exemple de Rovalo (1966) 244, nous les qualifions de «wisigothiques» par opposition aux hymnes antérieures de l'époque «hispano-romaine» et, celles, postérieures, de l'époque mozarabe. L'utilisation du terme «mozarabe» au sens large est très discutée, cf. Castro Sánchez / García Ruiz (2014) 16 avec bibl. n. 6. 


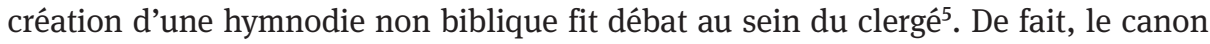
$13 \mathrm{du} \mathrm{IV} \mathrm{V}^{\mathrm{e}}$ Concile de Tolède en 633, inspiré et présidé par Isidore de Séville, laisse entrevoir l'attitude d'un clergé hostile aux hymnes composés en dehors des Écritures, alors que la composition d'hymnes postcanoniques était en plein essor :

[...] il est de notoriété publique que quelques hymnes ont été composés par le zèle des hommes pour la louange de Dieu, mais aussi pour les triomphes des Apôtres et des martyrs, tels ceux qu'ont produits les très bienheureux docteurs Hilaire et Ambroise - et pourtant certains les réprouvent nommément pour la raison qu'ils ne procèdent pas des saintes Écritures canoniques ou de la tradition des Apôtres - ; qu'ils rejettent donc aussi ce fameux hymne, composé par des hommes, que nous chantons chaque jour dans les offices publics et privés, à la fin de tous les Psaumes [...] et toute la suite que composèrent des docteurs de l'Église $[. . .]^{6}$

Sans entrer dans le détail des arguments scripturaires et patristiques invoqués par Isidore pour justifier l'hymnodie «humaine ${ }^{7}$, on notera, à la fin du canon, l'allusion aux compositions actuelles qui devaient, à l'exemple des hymnes d'Hilaire et d'Ambroise affirmant et défendant la foi de Nicée, être «les instruments efficaces d'une pédagogie de la foi ${ }^{8}$ ».

La manière dont Isidore inscrit de la sorte l'hymnodie de son époque dans la continuité des laudes des grands évêques $\mathrm{du} \mathrm{IV}^{\mathrm{e}}$ siècle pose la question de la place de la doctrine dans ces poèmes d'un autre temps. De fait, le contexte politico-religieux n'était plus le même qu'au $\mathrm{IV}^{\mathrm{e}}$ siècle, où l'essentiel de la foi était sans cesse menacé par des hérésies diverses : même si un sentiment d'insécurité a pu persister un temps, surtout dans le clergé, tout le peuple wisigoth s'était solennellement converti au catholicisme lors du III $^{\mathrm{e}}$ Concile de Tolède en 589 . Quelle forme prend, dès lors, la doctrine dans ces hymnes, a fortiori dans les hymnes du temporal et ceux pour des occasions variées?

S’il n'était plus nécessaire de barrer la route à l'hérésie arienne, on verra qu'une partie de l'hymnodie ecclésiale continuait, comme chez les grands évêques hymnographes, d'être le support d'une foi authentique, et a abouti à des hymnes d'une élaboration doctrinale remarquable, conformément à la liturgie de l'époque. On verra cependant qu'en fait de doctrine, le contenu de plusieurs hymnes se limite singulièrement à une mise en vers plus ou moins littérale de la Bible, dont on examinera les modalités caractéristiques.

5 Voir, sur ce débat, Fontaine (1999).

6 Trad. Fontaine (1999) 387.

7 Ils sont au centre de Fontaine (1999), qui emploie l'expression citée à la p. 391.

8 Fontaine (1999) 394. 


\section{L'élaboration doctrinale dans les hymnes de facture ambrosienne}

Plusieurs hymnes de l'ancienne liturgie hispanique répondent à l'exigence proprement doctrinale qui présida, selon Isidore, à la création des hymnes postcanoniques. Comme chez Ambroise ${ }^{9}$, ce contenu doctrinal est particulièrement élaboré dans les hymnes du temporal, dont les festivités, de l'Incarnation à la Passion, de la Résurrection à la Pentecôte, étaient propices à la célébration du mystère du Christ et de sa mission.

Les exemples les plus intéressants sont significativement constitués par des hymnes de facture ambrosienne, i.e. des hymnes composés de quatrains de dimètres iambiques, même s'il s'agit, comme on l'a rappelé, d'hymnes rythmiques et non quantitatifs, qui recherchent suivant une évolution médiévale la rime ; les strophes sont souvent au nombre du huit, mais pas forcément ${ }^{10}$. En raison des limites qui nous sont imparties, notre étude se limitera à un hymne dédié à la célébration de l'Adventus (« venue, avènement»), en référence à la période avant Noël pendant laquelle les fidèles se préparent à célébrer l'arrivée du Christ : d'après ses premiers mots, Gaudete, flores martirum ${ }^{11}$. L'examen de son contenu doctrinal sera l'occasion d'une confrontation avec la manière d'Ambroise, en particulier du point de vue du choix des sources et, plus largement, des traditions suivies en son temps par le Milanais. On verra ainsi que, si ces hymnes hispaniques trouvent, comme les hymnes ambrosiens, leur point de départ dans la Bible, l'invention lyrique suit une voie différente, en phase avec les orientations liturgiques de l'époque.

Comme l'attestent les titres transmis par la tradition manuscrite ${ }^{12}$, l'hymne Gaudete, flores martirum vise à célébrer le début de l'Avent, le 17 novembre, dont la date coïncide, dans le calendrier liturgique hispanique, avec l'anniversaire des saints Aciscle et Victoire, martyrisés à Cordoue en $303^{13}$. Vraisemblablement chanté lors des matines $^{14}$, il prend la forme d'un chant de réjouissance ${ }^{15}$ :

9 Voir, sur les hymnes d'Ambroise, Fontaine (1992).

10 Il s'agit d'un assouplissement fréquent qu'on trouve, dès la fin de l'Antiquité, dans des liturgies variées. En dépit de ses variations, le nombre de strophes reste toutefois limité aux $\mathrm{vI}^{\mathrm{e}}$-vII ${ }^{\mathrm{e}} \mathrm{s}$., avec une prédominance des poèmes de six ou huit strophes, cf. Valcárcel Martínez (2005) 146.

11 Il s'agit de l'hymn. 1 dans l'édition de J. Castro Sánchez.

12 Castro Sánchez (2010) retient l'intitulé In adventu Domini. In festo sancti Aciscli et sociorum. 13 Voir, sur cette conjonction du temporal et du sanctoral dès l'époque wisigothique, Rovalo (1966); en général sur la célébration de l'Avent dans l'ancienne liturgie hispanique, Ferro Calvo (1972). Suivant l'une des tendances étudiées par Rovalo (1966), les saints célébrés le 17 novembre sont évoqués de manière très générale (aucun d'eux n'est nommément cité), à égalité avec le thème de l'Avent. Cette conjonction étroite du temporal et du sanctoral se retrouve dans l'Officium Inquoante Adventu Domini du Misticus Toledano 35.4 pour lequel fut sans doute composé cet hymne au viI ${ }^{\mathrm{e}}$ s., cf. Rovalo (1966), 260-261 et 291. - Voir en général, sur cette relation étroite entre temporal / sanctoral (avec un enrichissement mutuel) qu'on observe à partir du début du viI ${ }^{\mathrm{e}}$ s., Janeras (1954). 
1

5

10

15

20

25
Gaudete flores martirum, salbete, plebes gentium, visum per astra mittite, sperate signum glorie.

Voces prophetarum sonant, venire Ihesum nuntiant, redemtionis previa, qua nos redemit gratia.

Hinc mane nostrum promicat et corda leta exestuant, cum vox fidelis personat prenuntiatrix glorie.

Tante salutis gaudium ! Quod est redemtum seculum, exceptionis inclitum abhinc ciamus canticum !

Adventus hic primus fuit, punire quo non seculum venit, sed ulcus tergere, salbando quod perierat.

At nunc secundus premonet adesse Xristum ianuis, sanctis coronas reddere celique regna pandere.

Eterna lux promittitur, sidusque salbans promitur; iam nos iubar prefulgidum ad ius uocet celestium.
Réjouissez-vous, fleurs des martyrs, salut, peuples des nations, portez votre regard vers les astres, espérez le signe de la gloire.

Les voix des prophètes résonnent, annoncent la venue de Jésus, prélude de la rédemption, de la grâce par laquelle il nous a rachetés.

Aussi, notre matin étincelle, et nos cœurs bouillonnent de joie, quand la voix de la Foi résonne, pré-annonciatrice de la gloire.

Joie d'un si grand salut !

Puisque le siècle a été racheté, élevons donc, pour l'accueillir, un cantique solennel!

Ce fut sa première venue, quand il vint non pour punir le siècle, mais pour effacer sa blessure, en sauvant ce qui était perdu.

Mais à présent la seconde prévient que le Christ se tient à la porte, qu'il rend aux Saints leur couronne et ouvre le royaume des cieux.

La lumière éternelle nous est promise, et l'astre salvateur apparaît ; déjà son éclat resplendissant nous appelle à la justice céleste.

14 Cf. le titre notamment donné par le manuscrit M (Madrid, Biblioteca Nacional 10001, $\mathrm{IX}^{\mathrm{e}}-\mathrm{XI}^{\mathrm{e}} \mathrm{s}$.), que semble corroborer mane au v. 9 : Ymnus de adventu domini et sanctorum festivitate ter mixtus qui in matutinum dicendus est quando adventus domini incipitur. L'office in matutinum correspondait manifestement à nos laudes même si, dans certains cas, il semble avoir inclus nos matines, cf. Fernández Alonso (1955), 338-339.

15 En accord avec les trois objets de la célébration présentés comme tels dans le titre du ms. M (l'Avent, les martyrs et le matin, cf. l'expression ter mixtus dans le titre cit. n. 14), sont ainsi évoqués, dans l'ordre du texte, la symbolique psalmique attachée à ce moment de la journée (mane... corda leta, v. 9-10, cf. Ps 29, 6), la joie liée à la naissance du Christ (gaudium, v. 13) et le bonheur céleste promis aux martyrs (beato cetui, v. 36). Deux des injonctions qui ponctuent le texte invitent par ailleurs expressément à la réjouissance et, partant, au chant de louange : gaudete (v. 1), gaudium (v. 13) et abhinc ciamus canticum (v. 16), où l'adverbe abhinc explicite la relation de cause à effet entre le gaudium du v. 13 et le canticum du v. 16. 
Te, Xriste, solum querimus videre sicut es Deus, ut leta nos hec visio evellat omni tartaro,

Quo dum redemtor veneris cum candidato martyrum globo, adunes celibi nos tunc beato cetui.

Deo patri sit gloria eiusque soli filio cum spiritu paraclito et nunc et in perpetuum.
Nous voulons seulement, ô Christ, te voir comme tu es, Dieu, afin que cette heureuse vision nous arrache entièrement au tartare,

pour que le jour où tu viendras, rédempteur, avec la foule blanche des martyrs, tu nous adjoignes à cette sainte assemblée bienheureuse.

Gloire à Dieu le Père et à son Fils unique, avec l'Esprit Paraclet, maintenant et pour toujours.

La venue du Christ est présentée de manière très abstraite, ainsi qu'en témoigne l'absence de toute référence narrative ou descriptive à l'incarnation ou à la nativité par exemple. Elle est évoquée à travers un dense réseau d'images lumineuses qui se prêtent, d'une part, à une lecture référentielle, à travers l'allusion à la lumière du matin au v. 9 (mane... promicat, où nostrum invite toutefois d'emblée à dépasser ce premier niveau et à interpréter mane comme une image du Christ ${ }^{16}$ ) ; d'autre part, surtout à une interprétation symbolique, suivant l'orientation suggérée au v. 4 par l'expression signum glorie annonçant les prophéties vétérotestamentaires évoquées dans la $\S 2$. De fait, cette lumière est celle de toute théophanie ; elle est aussi, dans la continuité du khabod hébraïque, une manifestation de la gloire divine et, en référence à Jn 8, 12, une figure du Christ-lumière ainsi qu'une préfiguration de la lumière éternelle.

Cette représentation imagée concourt à la dimension contemplative de l'hymne. L'assemblée en prière, comprise dans la première personne du pluriel, est invitée dès l'injonction du v. 3, en écho à Is 40.26 et 51.6, à tourner ses yeux vers le ciel ; la prière de demande des v. 29 sqq., qui émane directement des orants, lui fait écho à la fin de l'hymne (Te, Xriste, solum querimus / videre sicut es Deus...). L'expression Te... videre sicut es Deus, qui permet d'assimiler le Christ au Père, est notable par sa charge doctrinale ${ }^{17}$. Par-delà l'égalité des deux personnes, cette contemplation est cependant surtout l'occasion d'une méditation sur le sens de l'adventus Domini, dans la perspective non seulement de la venue de Jésus, mais aussi de la parousie du Christ.

Comme cela ressort nettement de la division, la célébration de la première venue du Fils de Dieu est en effet étroitement liée à l'attente de sa seconde venue à la fin

16 Castro Sánchez / García Ruiz (2014), 102d.

17 Elle est cependant moindre que dans l'hymn. 3 Xristi caterba clamitet composé à la même époque pour le premier dimanche de l'Avent où la même idée est exprimée en des termes d'une densité théologique remarquable (mais caractéristique de la liturgie hispanique, cf. infra), cf. les v. 13-16 A matre natus tempore, / sed sempiternus a patre / duabus in substantiis / persona sola est numinis. 
des temps. Dès la strophe introductive, le signum glorie du (v. 4) se réfère de manière ambivalente à l'un et l'autre de ces adventus, avant que, dans un parfait équilibre des masses, un groupe de quatre strophes soit consacré à l'adventus... primus (v. 17, cf. $\S 2-5$ ), puis au secundus (v. 21, cf. § 6-9).

Le premier mouvement est centré sur la rédemption préparée par la venue de Jésus (redemtionis previa, v. 7). Cette dernière est signifiée en des termes d'origine néotestamentaire, comme gratia et redemptio ${ }^{18}$ ou le participe redemtum (seculum) qui évoque plusieurs occurrences bibliques du verbe simple ou composé $\mathrm{e}^{19}$. On notera également, dans les v. 18-20, l'écho à Jn 12.47 et la citation quasi littérale de Mt 18.11 :

v. $18-20$ :

punire quo non seculum venit, sed ulcus tergere, salbando quod perierat.
Jn 12.47 non enim veni ut iudicem mundum sed ut salvificem mundum

Mt 18.11 venit enim Filius hominis salvare quod perierat (cf. Lc 19, 10)

Dans le second mouvement dominent, de manière significative, les visions empruntées à l'Apocalypse. Celles de la $\S 6$ donnent à voir l'accueil réservé aux saints par le Christ (avec, en référence à 2 Tm 4.8, l'octroi de la couronne) :

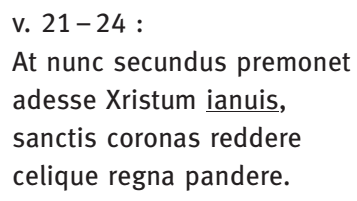

Ap 3.20 ecce sto ad ostium et pulso si quis audierit vocem meam et aperuerit ianuam... Ap 19.11 et vidi caelum apertum...

La $\S 7$ s'organise autour de deux images lumineuses. Celle de la lumière éternelle est commune à l'évangile de Jean $(8,12 ; 12,46)$ et à l'Apocalypse $(21.23-24 ; 22.5)$; quant à celle de l'astre salvateur, elle évoque l'étoile du matin d'Apocalypse 2.28 et 22.16. Elles préparent la prière de demande des $\S 8-9$ où les orants répondent à l'appel du iubar prefulgidum du v. 27 en priant le Christ de jouir à leur tour de cette vision bienheureuse lors de son avènement, au centre de la $\S 9$, fondée sur l'amalgame de plusieurs images empruntées à l’Apocalypse :

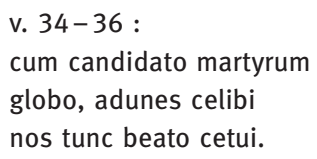

Ap 7.9 vidi turbam magnam... amicti stolas albas
et palmae in minibus eorum Ap 20.4 et vidi... et animas decollatorum propter testimonium lesu et propter verbum Dei Ap 21.3 ... ecce tabernaculum Dei cum hominibus et habitabit cum eis et ipsi populus eius erunt et ipse

Deus cum eis erit eorum Deus

18 Cf. p. ex. Rm 3.23-24 (omnes enim peccaverunt ... justificati gratis per gratiam ipsius per redemptionem quae est in Christo Iesu).

19 Cf. 1 Co 6.20 ; 7.23 ; 1 P 1.18. L'expression est redemtum seculum a joui d'une grande fortune dans l'hymnodie, cf. infra n. 33. 
Cette évocation de la venue du Christ rédempteur entouré de la troupe blanche des martyrs permet, en écho à l'invocation liminaire des flores martirum, de rattacher la célébration du début de l'Avent à celle des saints fêtés le même jour dans le calendrier liturgique hispanique. Il est fort probable que la volonté de chanter conjointement la venue du Christ et les martyrs mis à l'honneur le premier jour de l'Avent ait déterminé le choix de la structure, avec la mise en parallèle du primus et du secundus adventus. Ce dernier trouve toutefois son origine dans une double tradition digne d'intérêt pour notre propos, car elle est caractéristique des hymnes doctrinaux de l'ancienne liturgie hispanique.

Inusité par ailleurs dans le genre poétique ${ }^{20}$, le concept de primus et secundus adventus connut en effet une grande fortune chez les Pères latins ${ }^{21}$, à partir desquels il fut vraisemblablement intégré, dès l'époque wisigothique, aux textes de l'office. Bien que les livres liturgiques hispaniques soient tous relativement tardifs ${ }^{22}$, l'Oracional visigotico édité par J. Vives atteste en effet l'utilisation de cette expression d'origine patristique dans les prières employées lors de l'office de Adventu Domini ${ }^{23}$; il n'est que de songer à cette benedictio prononcée lors des vêpres du $5^{\mathrm{e}}$ samedi où l'évocation de la venue du Christ apparaît, comme dans l'hymne Gaudete, flores martirum, une source de joie :

Christus Dei filius, quem venturum in proximo sustinetis, proximitate sui adventus emundet corda vestra a cunctis mundanae vitae inlecebris. Ipsum quoque quem iudicem venturum metuitis, liberatorem indulgentissimum sentiatis. Quo omnes, qui hic primi adventus sui gaudia devote inquolitis, secundi adventus sui premia capiatis. ${ }^{24}$

Ce type d'emprunts est habituel dans les textes de l'ancienne liturgie hispanique, qui porte l'empreinte marquée des Pères ${ }^{25}$; les hymnes ne font pas exception.

Comme en témoigne la densité des références relevées par J. Castro Sánchez dans son apparat critique, les expressions d'origine patristique sont nombreuses dans

20 En dehors justement de l'hymne wisigothique Xristi caterba clamitet évoqué dans la n. 17 (cf. le v. 29 où l'adjectif secundus se réfère, en écho au v. 28, à l'adventus... secundus).

21 Pour nous limiter au témoignage très proche, d'un point de vue spatio-temporel, d'Isidore, cf. fid. I.61.2 sqq.

22 Cf. le recensement de Díaz y Díaz (1965b) 109-164 («Los textos de la antigua liturgia hispanica Fuentes para su estudio »), d'où ressort la rareté des sources liturgiques de l'époque wisigothique. 23 Voir, sur cette utilisation, Vives (1946) XV ; sur sa datation, que Vives situe à la fin du vil ${ }^{\mathrm{e}} \mathrm{s}$. ou au début du viII ${ }^{\mathrm{e}}$ s., en tout cas avant 711, Vives (1946) XXXII-XXXIv.

24 Vives (1946), Oratio 35, cf. 0.3 .45 ; le motif de la joie revient également de manière récurrente, cf. par exemple les Orationes 30, 36, 44, 53, etc. L'expression se retrouve dans les livres liturgiques postérieurs édités par Férotin (1912), cf. entre autres ce passage de la messe du $3^{\text {e }}$ dimanche de l'Avent : Quosque prospicis adventum olim mystice peracte Incarnationis tue nunc promtissima devotione suscipere, hos facito in secundum iudicii tui adventum ad te exsultantibus animis pervenire, atque in amenitate paradisi iucundaluros induce (c. 15-16).

25 Cf. sur cette influence plus marquée dans la liturgie hispanique que dans les autres liturgies antiques, Díaz y Díaz (1965b), 59-62. 
l'hymne Gaudete, flores martirum. Il peut s'agir d'expressions relativement communes chez les Pères, comme l'expression voces prophetarum (v. 5) dont on trouve plusieurs attestations en référence aux prophéties annonçant la venue de Jésus ${ }^{26}$; d'autres sont moins usuelles, à l'exemple du tour plebes gentium qui trouve probablement sa source dans l'interprétation allégorique du Cantique des Cantiques de Grégoire d'Elvire ${ }^{27}$.

L'influence de la tradition liturgique n'est pas moins nette, même si elle est difficile à évaluer en raison de la rareté des témoins wisigothiques ; on peut en juger grâce aux exemples suivants - tous personnels - empruntés à l'Oracional visigotico édité par J. Vives ${ }^{28}$ ou, en raison de leur résonance liturgique caractéristique, peutêtre déjà wisigothique, à des manuscrits postérieurs édités par M. Férotin ${ }^{29}$ :

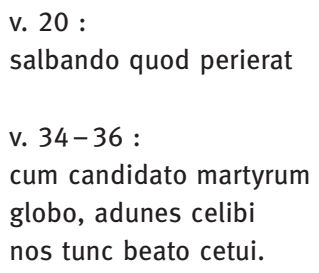

Oratio 9 Ex Syon Deus veniens, ab impietatibus vestris eripiat vos. [...] Tales etiam vos redimendo statuat, quales iudicando non puniat.

Oratio 63 Christe Dei filius, cum quo sanctorum milia ad iudicium venient, sanctorum vos cetibus coronandos adsociet, cf. Oratio 127 ... sanctorum mereamur et congregationi et cetibus interesse.

Ferotin c. 490 Et que iam suo sunt candidate martyrio Ferotin c. 576 iste himnus, Domine, Martirum tuorum candidatus laudat exercitus

Ferotin c. 875 et secunde mortis calcata severitate sanctorum cetui mereamur obtinere societatem.

Les emplois liturgiques de caelebs (celibi, v. 35) dans le sens de "céleste, saint»" tendent dans le même sens. Cette double filiation, qui concourt, sur fond de réminiscences bibliques, à l'élaboration doctrinale du poème, charge sans jamais entraver l'harmonie de son mouvement, l'expression lyrique d'une inspiration pleinement religieuse, là où les hymnes d'Ambroise portent l'empreinte de la tradition poétique profane, en particulier virgilienne ${ }^{31}$. Rares sont en effet, dans l'hymnodie hispanique, les réminiscences poétiques et, le cas échéant, elles se limitent aux

26 Cf. p. ex. Arnobe In Psalm. 31 Mundus [...] clamavit semper per voces prophetarum, ut veniret qui salvaret eum ; 118.81-176 Sciunt enim et intellegunt voces prophetarum, in quibus verba tua pollicentur nostri redemptoris adventum ; Grégoire le Grand, Moral. 30.10.36 ... hoc idem tempus incarnationis domini ante prophetarum voces cognitum non fuit...

27 Cf. en particulier ce passage du livre II, où l'on trouve une attestation remarquable de cette expression très rare par ailleurs : plebes gentium ante adventum Christi sub iugo et potestate diaboli subditas esse dicebat (CPL 547, 1. 190).

28 Cf. en outre le parallèle qu'on peut faire entre les vers de l'hymne 3 cités dans la n. 16 et le début de l'Oratio 10 Christe Dei filius, qui a patre sine tempore natus, per virginem in tempore nasciturus... 29 Férotin (1912).

30 Cf. Castro Sánchez (2010) 735 et Castro Sánchez / García Ruiz (2014) 102 (h).

31 Cf. Fontaine (1992) 26 ; en général, sur les modèles poétiques classiques des hymnes d'Ambroise, Fontaine (1974/75). 
suggestions de la tradition hymnique chrétienne. Outre la reprise de la structure métrique caractéristique des hymnes ambrosiens, l'hymne Gaudete, flores martirum ne compte ainsi que deux emprunts manifestes à l'œuvre poétique de Prudence qui jouit, à la fois en raison de sa spiritualité infuse et de son origine espagnole, d'une grande fortune dans la liturgie hispanique ${ }^{32}$ :
v. 1 Gaudete, flores martirum
v. 12 prenuntiatrix glorie
Prudence, Cath. 12.125 Salvete flores martyrum
Prudence, Perist. 2.30 praenuntiatrix gloriae

L'unique autre parallèle poétique concerne un autre hymne hispanique ${ }^{33}$. Mais outre la délicate question de la filiation qui relie ces textes, il s'agit alors d'un cas particulier d'imitation «interne», peu ou prou semblable à celui qui rattache ces hymnes aux autres textes liturgiques, dans un réseau d'une densité remarquable.

\section{Les réécritures de la Bible : modalités et enjeux}

Nombreux sont, à côté de ces hymnes doctrinaux, les pièces qui empruntent tout ou partie de leur matière aux Écritures. Il s'agit, pour les raisons qu'on a vues précédemment, principalement d'hymnes du temporal ; mais la célébration isolée de certaines heures, en particulier le chant du coq, et celle de plusieurs saints bibliques (comme Jean-Baptiste ou Étienne) étaient propices à la reprise de péricopes bibliques, souvent très fidèles à la lettre du texte ${ }^{34}$. Pour mesurer les enjeux de la réécriture, notre attention ira à deux pièces qui illustrent chacune un traitement différent du texte biblique, le premier narratif, le second exégétique ${ }^{35}$.

\section{a) Les paraphrases narratives}

Selon un usage largement répandu dans l'hymnodie hispanique, en particulier dans les pièces du sanctoral, plusieurs de ces hymnes d'inspiration biblique prennent une forme narrative suivant la division tripartite invocatio - narratio - peroratio (avec

32 Voir à ce sujet Urlacher-Becht (2012).

33 Cf. hymn. 1.14 quod est redemtum seculum et 83.11 a quo redemtum seculum.

34 La part de la paraphrase biblique est très importante dans les hymnes suivants, dont la datation est particulièrement problématique dès lors qu'ils ne sont conservés que dans les hymnaires d'éditions imprimées : hymn. 27 (Carême) ; 25.30 et 41 (Pentecôte - avec la focalisation sur le chant du coq dans l'hymn. 30) ; 81 (Purification de la Vierge Marie) ; 132 (Saints Innocents) ; 134-135 (Jean Baptiste) ; 171 (Saint Étienne). Sur ce procédé qui serait caractéristique de l'ancienne hymnodie hispanique, cf. Messenger (1946) 160-161. L'hymn. 30, qui fait partie de la même série que l'hymn. 25 évoqué infra, fait partie des pièces caractéristiques de l'époque wisigothique étudiées par Valcárcel Martínez (2005).

35 Voir, sur cette distinction entre paraphrases narratives et exégétiques, Charlet (1985) 631 sqq. 
doxologie) ${ }^{36}$. Leurs auteurs anonymes s'inscrivent dans le sillage de poètes illustres dont les amples pièces avaient été intégrées, parfois de manière fragmentaire, à la liturgie hispanique : on pense notamment aux hymnes du Cathemerinon, tous construits autour d'une paraphrase biblique et dont la diversité métrique a probablement influencé celle des pièces qui nous occupent ; d'autre part, à l'hymne alphabétique de Sédulius relatant la vie du Christ de sa naissance à l'Ascension. ${ }^{37}$ Comme ce dernier, la plupart de ces poèmes consistent de prime abord en une simple réécriture lyrique d'une ou de plusieurs péricopes bibliques, où la multiplication des citations quasi littérales semble fortement limiter la part de la création poétique. L'étude détaillée de l'hymne Auctor luminis, filius virginis ${ }^{38}$ montrera cependant qu'il n'en est rien.

Cet hymne, composé pour l'office des vêpres du deuxième dimanche du Carême, est constitué de 8 strophes saphiques ${ }^{39}$. Par son fond et sa forme, il s'apparente à une simple mise en vers du texte évangélique du jour : Jean 9 qui relate la guérison miraculeuse d'un aveugle né ${ }^{40}$.

1 Auctor luminis, filius virginis paterque matris filiusque patris, qui ceco sedenti stipite ${ }^{41}$ petenti lumen dedisti.

5 Sedensque cecus tenebris oppressus, quem voluerat sic nasceret cecus, in quem virtus sua omnibus credentis comprobaretur.

Gaudetque cecus lumine recepto, 10 quem visitabit medicus exelsus a patre dilectus, ut ablueretur mundi delictum.
Auteur de la lumière, fils d'une vierge, Père de ta mère et Fils du Père, qui à l'aveugle assis, mendiant de l'argent, as donné la lumière.

Assis, l'aveugle était oppressé par les ténèbres, Dieu avait voulu qu'il naquît aveugle, afin qu'en lui tous les croyants reconnaissent son pouvoir.

Et l'aveugle se réjouit d'avoir reçu la lumière, lui qu'a visité le médecin suprême, choisi par le Père pour enlever le péché du monde.

36 Cf. Díaz y Díaz (1980) 68-70.

37 Voir, sur la «création poétique» dans le Cathemerinon, Charlet (1982) et, sur la réception de Prudence dans la liturgie hispanique, Urlacher-Becht (2012) 77-79. Tout comme de nombreux hymnes anonymes, plusieurs pièces de poètes connus furent intégrées à la liturgie hispanique, cf. dernièrement dans Castro Sánchez / García Ruiz (2014) 21, la liste invariablement reprise d'une édition à l'autre (avec toutefois des erreurs, les hymnes d'Ennode de Pavie n'ayant jamais été chantés en Hispanie, cf. Urlacher-Becht 2014269 n. 14).

38 Hymn. 25 dans l'édition de Castro Sánchez.

39 Voir, sur cette structure métrique particulièrement goûtée aux $\mathrm{VII}^{\mathrm{e}}-\mathrm{VIII}^{\mathrm{e}}$ s., Valcárcel Martínez (2005), 150, avec une analyse sommaire de l'hymn. 25 dans les p. 150 sqq. Ce dernier est daté du viI ${ }^{\mathrm{e}}$ s. par Castro Sánchez / García Ruiz (2014) 172.

40 Le texte de tous les offices du $2^{\mathrm{e}}$ dimanche du Carême est conservé en intégralité dans le Liber misticus de Cuaresma y Pascua (Cod. Toledo, Bibl. Capit. 35.5) édité par Janini (1980) 11-19: la lecture de Jn 9.1-38 Ad missam est spécifiée à la p. 17 ; sur ce type de livre liturgique, cf. Pinell (1954). 41 stipite = stipem, cf. Castro Sánchez (2010) 739. 
Plebs Ebreorum zelumque dixerunt: "parentes eius veniant»; perquisierunt: «qui cecus est natus, cuius virtute est inluminatus?».

Parentes autem Ebreorum fatent: «ex utero matris cecus fuit natus, etatem habet, ipsum interrogate, dicat veritatem ».

Confidet cecus qui fuerat factus: «ad me accessit prophetaque magnus, cuius virtute oculo et corde sum inluminatus.

25 Fecitque lutum de sancto sputo, tegmen oculorum linuit, a luto iussit labari Siloe piscina, me clarificavit».

Gloria patri, trino sempiterno, 30 gloria Xristo spirituque sancto, predicet trinum pie voce nomen omne per evum.
La foule des Hébreux dit avec jalousie : «Que ses parents viennent »; ils les interrogèrent :

«Lui qui est né aveugle, par le pouvoir de qui a-t-il reçu la lumière ?»

Mais ses parents, des Hébreux, déclarèrent : «Il est né aveugle du ventre de sa mère ; il est assez grand, interrogez-le lui-même, qu'il dise la vérité.»

Celui qui était né aveugle confessa : «Un grand prophète s'est approché de moi : par son pouvoir, mes yeux et mon cœur furent illuminés.»

De sa sainte salive, il fit de la boue, l'appliqua au voile de mes yeux, et m'ordonna de laver la boue à la piscine de siloé ; ainsi il m'éclaira.»

Gloire au Père, à la Trinité éternelle, gloire au Christ et à l'Esprit Saint ; que d'une voix pieuse le nom ${ }^{42}$ trine soit célébré à tout jamais.

La narration occupe la plus grande partie du poème (§ 2 à 7 vs une seule strophe pour l'invocation liminaire et la doxologie finale). Comme en témoignent les nombreux emprunts textuels relevés dans le tableau suivant, elle s’inscrit dans la continuité directe du récit de Jn 9.

v. 3 qui ceco sedenti stipite petenti

6 quem voluerat sic nasceret cecus, in quem virtus sua omnibus credentis comprobaretur.

13 Plebs Ebreorum zelumque dixerunt: "parentes eius veniant»; perquisierunt: «qui cecus est natus, cuius virtute est inluminatus?».

17 Parentes autem Ebreorum fatent: "ex utero matris cecus fuit natus,
Jn 9.8 mendicus erat... nonne hic est qui sedebat et mendicabat

Jn 9.1 hominem caecum a nativitate (cf. In 9, 19-20)

Jn 9.3 ... ut manifestetur opera Dei in illo

In 9.18-19 non crediderunt ergo ludaei de illo quia caecus fuisset et vidisset donec vocaverunt parentes eius qui viderat et interrogaverunt eos dicentes hic est filius vester quem vos dicitis quia caecus natus est quomodo ergo nunc videt

In 9.20 responderunt eis parentes eius et dixerunt... quia caecus natus est

42 Par nomen, il faut entendre "personne», cf. Castro Sánchez / García Ruiz (2014) 183 (e). 
etatem habet, ipsum interrogate, dicat veritatem».

22

«ad me accessit prophetaque magnus

25 Fecitque lutum de sancto sputo, tegmen oculorum linuit, a luto iussit labari Siloe piscina, me clarificavit».
In 9.21 ipsum interrogate aetatem habet ipse de se loquatur (cf. Jn 9.23)

Jn 9.17 quia propheta est

In 9.6 haec cum dixisset expuit in terram et fecit lutum ex sputo et linuit lutum super oculos eius et dixit ei vade lava in natatoria Siloae quod interpretatur Missus abiit ergo et lavit et venit videns (cf. $9.11 ; 15$ )

De fait, les variantes observées, à l'instar de Siloe piscina (v. 27) vs Jn 9.6 in natatoria Siloae, sont manifestement dues à des considérations métriques. L'élaboration narrative n'est pas nulle pour autant. On observe une réduction substantielle de l'hypotexte, qui passe notamment par la suppression des redites dues aux interrogatoires répétés et la réduction à l'essentiel du cheminement conduisant l'ex-aveugle vers la foi ; elle tient aussi à la réorganisation de certains éléments (comme le fait de présenter d'emblée l'aveugle comme un mendiant : v. 3, cf. Jn 9.8), qui permet au récit de gagner en précision et en fluidité. Mais la narration n'est pas seulement condensée : elle fait l'objet d'une dramatisation à travers l'utilisation répétée du discours direct qui rend la scène à la fois plus vivante et plus aisée à se représenter ${ }^{43}$. Par-delà la question de la représentation, le resserrement opéré permet aussi et surtout de valoriser divers éléments suggérant une interprétation allégorique qui approfondit celle qui est donnée dans Jn 9.35-4144.

À la fin du récit, l'aveugle présente en effet sa guérison miraculeuse comme le résultat d'une intervention divine qui lui a permis de reconnaître le fils de Dieu et, ce faisant, d'accéder à une vision spirituelle de la foi ; il devient ainsi l'allégorie d'une humanité aveuglée par ses péchés, à laquelle le Christ, lumière du monde, est venu «ouvrir les yeux». Cette lecture est clairement suggérée aux v. 7-8 où nous comprenons, en relation avec l'idée de preuve (comprobaretur), que tous sont portés à croire grâce à l'exemple de manifestation de la puissance (virtus) de Dieu au centre du récit. L'idée de virtus est répétée à deux reprises dans la suite du texte, en relation avec la forme verbale inluminatus est dont on trouve, dans la tradition patristique, plusieurs occurrences pour signifier, au sens propre, la vue recouvrée, au sens figuré, l'illumination de la foi ${ }^{45}$. De fait, l'auteur de l'hymne joue constamment sur ces deux niveaux de signification, les juifs s'interrogeant, aux v. 14-15, sur les circonstances

43 Cet aspect de la réécriture a été noté par Valcárcel Martínez (2005) 152.

44 Non repris dans l'hymne, alors que la lecture de Jn 9 s'arrêtait au verset 38.

45 Parmi les nombreuses occurrences de l'expression caecum ou caecos illuminare, cf. notamment Augustin, serm. 136C (REAug 24, p. 89, 1. 15), en référence à Jn $9:$ : [...] huius dominici quo illuminatus est caecus [...]. Le verbe illuminare, non attesté dans la Bible, est également usuel, sous l'influence des Pères, dans la tradition liturgique, cf. dès l'époque wisigothique cette benedictio prononcée lors du Carême : Christus Dei filius labores vestros propitiatus aspiciat, et cordis vestri tenebras inluminando submoveat (Oratio 536, ed. Vives 1946). 
de la guérison miraculeuse, tandis que l'ex-aveugle reconnaît, aux v. 23-24, avoir accédé non seulement à la vue, mais aussi à la vision spirituelle de la foi (oculo et corde). Par-delà cette exégèse traditionnelle, induite par le texte de Jean, le traitement de la dichotomie ténèbres/lumière répond cependant aussi aux exigences de la célébration liturgique. Comme on l'a dit, cet hymne fut composé pour les vêpres du $2^{\mathrm{e}}$ dimanche du Carême. Or on sait, depuis Ambroise, la place du jeu de l'ombre et de la lumière dans les hymnes des heures, en lien avec la symbolique johannique du Christ «lumière». Quant au Carême, il prépare à la fête pascale, traditionnellement présentée comme «un jour plus lumineux que les autres [...] ; un jour qui ne connaîtra pas de déclin ${ }^{46}$. La symbolique lumineuse est donc particulièrement bienvenue dans ce contexte, auquel se réfère l'allusion (absente en tant que telle de Jn 9) à la Rédemption aux v. 10-11 (ut ablueretur / mundi delictum).

L'hymne n'est donc pas dénué de toute portée doctrinale et prend même, dans les v. 1-2 et dans la strophe finale, un tour théologique qui inscrit le récit dans un cadre orthodoxe. La doxologie finale n'est guère originale. Un grand nombre des hymnes hispaniques s'achèvent, conformément au modèle ambrosien, par une doxologie trinitaire détachée de l'hymne et, de fait, souvent commune à plusieurs pièces ${ }^{47}$. L'invocation liminaire est, en revanche, étroitement liée à l'objet de la narration ${ }^{48}$. L'un des enjeux du récit de Jn 9 concerne en effet l'identité de Jésus, que les juifs refusent de reconnaître comme le fils de Dieu, tandis que la connaissance de l'ex-aveugle gagne, au fil du récit, en profondeur : d'abord un anonyme, Jésus devient pour lui un prophète (cf. propheta, v. 22), un homme de Dieu, et finalement le Fils de Dieu qu'il confesse dans la foi. Or la série de périphrases liminaires, qui jouent des paradoxes inhérents au dogme de l'incarnation, plongent d'emblée les orants dans le mystère de leur relation intime avec Dieu, tout en rendant le Christ, absent du récit, présent à leur conscience ${ }^{49}$. L'enjeu de l'hymne dépasse donc largement le récit : par-delà le développement du thème de la foi, le discernement de la nature divine du Christ est affirmé avec force par des orants appelés à se réjouir, comme l'aveugle dans la §5, de la venue de celui qui les lavera, par son sacrifice, du péché. Le lyrisme de la composition, fondé, par-delà le mètre, le rythme et la rime, sur les jeux de reprises et de variations, concourt grandement à cette ouverture des perspectives, et nous aide à saisir la portée

46 Cf. les références patristiques indiquées par H. Savon dans Fontaine (1992) 418-419 ; plus particulièrement sur l'importance du thème de la lumière lors de la célébration de Pâques dans l'ancienne liturgie hispanique, Fernández Alonso (1955) 361-362.

47 Cf. Castro Sánchez / García Ruiz (2014) 52-53.

$48 \mathrm{Cf}$. outre les éléments relevés dans la suite, l'introduction en filigrane du thème de la lumière à travers l'expression auctor luminis (v. 1).

49 L'expression paterque matris, qui présente de manière paradoxale le Christ comme le père de sa mère, apparaît dans l'Antiquité tardive, cf. l'étude de Mayer (1927) 66-72. Elle est attestée sous cette forme dans le symbole du $\mathrm{xI}^{\mathrm{e}}$ Concile de Tolède (675) où l'affirmation des deux naissances miraculeuses du Christ débouche sur la conclusion suivante : qui tamen secundum Deus est, creavit Mariam ; secundum quod homo, creatus est a Maria : ipse et Pater Matris, et Filius (§ 48, éd. Madoz 1938). 
spirituelle de cet hymne qui s’apparente, de prime abord, à une simple réécriture de l'Évangile du jour. On ajoutera que cette variation lyrique faisait d'autant moins «double emploi» avec le texte scripturaire qu'elle n'était pas destinée au même office. L'évangile de Jean était en effet lu lors de la «messe ${ }^{50}$, tandis que l'hymne était chanté lors des vêpres : cette distribution accrédite la thèse de deux utilisations complémentaires, orientée, pour la première, vers la catéchèse, pour la seconde, vers la contemplation et la célébration. Les hymnes narratifs se rapprochent en cela des hymnes doctrinaux ainsi que des hymnes exégétiques que nous considérerons in fine.

\section{b) Les paraphrases exégétiques}

Il existe, à côté des paraphrases narratives où divers éléments du récit recèlent les clés d'une interprétation allégorique, un second type de modalité de réécriture biblique où, suivant une tendance caractéristique de la poésie biblique tardive, la paraphrase proprement dite est suivie d'un développement exégétique donnant explicitement ses clés d'interprétation ${ }^{51}$. Les exemples les plus intéressants sont constitués par des hymnes pour des occasions variées, étrangers à la typologie ambrosienne, dont la liturgie hispanique recèle un nombre élevé reflétant sa richesse. De fait, dans ces hymnes composés pour des circonstances fort diverses, les références bibliques sont nombreuses, et sont parfois l'occasion de véritables réécritures dans des contextes moins attendus, sans équivalent dans les autres liturgies ${ }^{52}$. Notre attention ira, là encore, à un exemple emblématique de l'hymnodie wisigothique qui n'a guère retenu l'attention jusque-là : l'hymne Verus redemtor, Xriste, lumen luminis ${ }^{53}$.

Cet hymne, constitué de dix strophes de cinq trimètres iambiques rythmiques, était chanté lors de la consécration épiscopale, sur laquelle il constitue une source unique dans la liturgie hispanique ${ }^{54}$. Il fait pendant à l'hymne 191 composé pour l'office du matin, tandis que l'hymne 190 était utilisé ad vesperas. Son intérêt tient, après une strophe introductive d'une densité théologique remarquable, à l'ample réécriture proposée, dans les $\S 2$ à 5 , du passage de l'Exode consacré aux vêtements sacrés du premier grand prêtre, Aaron ; elle est suivie, dans les $\S 6-7$, d'une subtile exégèse d'Ex 28 qui débouche, dans les $\S 8-9$, sur la célébration de l'institution par le Christ des évêques à la tête de l’Église.

50 Cf. sur le sens controversé du titre «Missa», Pinell (1954) et Ramos (1964) 89-101; sur la «La misa del domingo », Fernández Alonso (1955) 328-330.

51 Voir, sur ce type de paraphrase, Charlet (1985) 641.

52 Cf. notamment, outre l'hymn. 191 étudié infra, la densité des hypotextes vétérotestamentaires dans l'hymn. 195 O verum regimen chanté lors du départ de l'armée du roi.

53 Il s'agit de l'hymn. 190. L'hymne daterait, d'après Díaz y Díaz (1986) 445-448, de la fin du règne de Chindaswinthe († 653).

54 La formule utilisée durant le cérémonial de la consécration des évêques n'est conservée dans aucun des nombreux manuscrits mozarabes, cf. Férotin (1904) 60-62. 
Verus redemtor, Xriste, lumen luminis, imago patris, splendor invisibilis, de patre natus antecedens tempora, ex matre vero prodiens in tempore, promissionis fedus inplens devite.

Tu es sacerdos, tu sacerdotes creas, tu principatum sortis huius floride Aaron dedisti, consecrator inclite, perfusione sacrosancti unguinis et veste sancta vultum eius preparans.

Sublime cuius in caput premittitur nitens olivum, mitra, auri lamina; stola decoris, vestimento poderis ambitur idem, quem sacratum nuntiat

15 inpressa vesti et colorum dignitas:

Coccus, iacinctus, torta bissus, purpura cuius dicati preferentes gloriam; vestis sacrate ora tintinnabulis erant corusca rite dependentibus, ut templum intrans audiretur pontifex.

Distincta gemmis pulcritudo pectoris affigebatur sub figuris optimis, zmaracdus illic, sardius, topatius, onicinus, carbunculus, ligurius, 25 saffirus, iaspis et nitens crisolitus.

Berillus inde seu acates fulgidi cum ametisto ordinis sui loco distinctionis iure illic positi dabant future gratie signaculum, 30 quo hec figure nuntiarent te Deum.

Huius dicate sacramentum ${ }^{55}$ gratie temtum per umbras transiendo seculi pervenit ad te, liberator obtime,
Vrai rédempteur, Christ, lumière de la lumière, image du Père, splendeur invisible, né du Père avant les temps, mais entré par sa mère dans le temps, accomplissant dûment le pacte promis.

Tu es prêtre, tu crées les prêtres ; la primauté de ce sort florissant, tu l'as donnée à Aaron, illustre consécrateur, en répandant le Saint Onguent et en préparant son visage par des vêtements sacrés.

En haut de sa tête sont appliquées l'huile brillante, la mitre, les lames d'or ; une étole d'apparat, une longue tunique le couvrent également, lui dont le sacre est annoncé

par la dignité des couleurs ornant ses vêtements :

écarlate, jacinthe, lin tordu et pourpre qui manifestaient la gloire de celui qui est consacré ; les clochettes suspendues rituellement aux pans du vêtement sacerdotal les rendaient étincelants, afin qu'on entendît le pontife entrer dans le temple.

Son pectoral se signalait par la beauté des pierres

qui y étaient fixées sous les meilleures figures : émeraude, sardoine, topaze, onyx, escarboucle, rubellite, saphir, jaspe et chrysolithe éclatante.

Puis, béryl et agates scintillantes, placés là avec l'améthyste au rang qui est le leur en guise de distinction, marquaient le signe de la grâce future, puisque ces figures t'annonçaient, Dieu.

Le mystère de la grâce de celui qui était ainsi consacré, retenu parmi les ombres du siècle en le traver-

55 Sur cet emploi de sacramentum dans le sens de mysterium, cf. Castro Sánchez / García Ruiz (2014) 509 (k). 
ut, que figure precinebant mistice, completa in te veritas ostenderet.

Hinc finis ipse legis ad iustitiam mundi reatum proximans detergere, oblatus ipse tu, oblator, consecrans in patris aram conlocatam victimam, hoc sacramento suscitans ecclesiam,

Caput cui factus et ducator previus duces in illam presulesque conlocans, quorum regatur instruenda legibus, ut erudita gratia pontificum vitare possit damna seva mortium.

Hinc te corona, Xriste, fratrum postulat, successionis clara huius gratia descendat in his, quos futuros preligis uni columbe preferendos principes, sant,

est parvenu à toi, libérateur par excellence, afin que la vérité, annoncée symboliquement par les figures, apparût accomplie en toi.

Puis toi-même, fin de la loi en vue de la justice, t’apprêtant à laver les fautes du monde, consacrant par ton propre sacrifice, sacrificateur, la victime placée sur l'autel du Père, tu as fait naître par ce mystère l’Église.

Tu as mis à sa tête un dignitaire montrant la voie, qui établit en elle les prélats comme guides; leurs lois la forment et la régissent afin qu'instruite par la grâce des pontifes, elle puisse éviter les cruels dommages de la mort.

Aussi, cette assemblée de frères te demande, Christ, que la grâce éclatante de cette succession descende sur ceux que tu as déjà choisis de présenter comme futurs chefs à l'unique colombe, qui leur confère la gloire de l'apostolat.

Suivant l'expression veste sancta du v. 10, la paraphrase des v. 6 à 30 est dédiée aux vêtements d'Aaron, sur la base d'Exode 28. Il s'agit, comme dans l'hymne 25, d'une réécriture très proche de la lettre du texte biblique, fondée sur de nombreux emprunts textuels qui sont ajustés aux exigences du mètre ${ }^{56}$. Elle s'accompagne là encore d'une nette réduction de l'hypotexte biblique qui passe, notamment, par la suppression des répétitions et la focalisation sur certains aspects, comme les insignes de la tête dans la §3 dédiée aux ornements vestimentaires d'Aaron. On notera par ailleurs l'effort d'organisation de la description, à travers des effets d'annonce et une distribution strophique éclairant sa progression ; on peut en juger grâce au tableau suivant, qui met en regard le texte des $\S 3$ à 6 avec les passages correspondants d'Ex 28 :

§ 3 Liste des vêtements Sublime cuius in caput premittitur (annoncée par vesta nitens olivum, mitra, auri lamina; sancta v. 10, cf. Ex 28.2) stola decoris, vestimento poderis ambitur idem, quem sacratum nuntiat inpressa uesti et colorum dignitas:
Ex 28.4 haec autem erunt vestimenta quae facient rationale et superumerale tunicam et lineam strictam cidarim et balteum facient

56 Cf. en part. la réorganisation des pierres énumérées dans les §5-6 dont rend compte la comparaison ci-dessous. 
§ 4 Liste des couleurs Coccus, iacinctus, torta bissus, purpura (annoncée par le dernier cuius dicati preferentes gloriam; vers de la §3) vestis sacrate ora tintinnabulis erant corusca rite dependentibus, ut templum intrans audiretur pontifex.
§ 5 et début de la $\S 6$ Liste des pierres incrustées sur le pectoral (pectoris, v. 21)
Distincta gemmis pulcritudo pectoris

affigebatur sub figuris optimis, zmaracdus illic, sardius, topatius, onicinus, carbunculus, ligurius, saffirus, iaspis et nitens crisolitus. Berillus inde seu acates fulgidi cum ametisto ordinis sui loco... vestimenta sancta Aaron fratri tuo et filiis eius ut sacerdotio fungantur mihi

Ex 28.5 accipientque aurum et hyacinthum et purpuram coccumque bis tinctum et byssum Ex 28.2 vestem sanctam ... in gloriam Ex 28.34-35 ita ut tintinabulum sit aureum... ut audiatur sonitus quando ingreditur et egreditur sanctuarium...

Ex 28.17-20 ponesque in eo quattuor ordines lapidum in primo versu erit lapis sardius et topazius et zmaragdus in secundo carbunculus sapphyrus et iaspis in tertio ligyrius achates et amethistus in quarto chrysolitus onychinus et berillus inclusi auro erunt per ordines $\underline{\text { suos }}$

Dans la continuité du texte biblique, cette description est en elle-même hautement symbolique. Plusieurs expressions suggèrent en effet, sur la base d'un symbolisme qui reste implicite, que les couleurs et les pierreries ornant les vêtements d'Aaron représentent sa sainteté (veste sancta vultum eius preparans v. 10 ; sacratum nuntiat v. 15, cf. sub figuris optimis v. 22) ${ }^{57}$. Mais surtout, ces ornements se prêtent, dans les $\S 6$ à 8, sur la base de l'Épitre aux Hébreux (chap. 7 à 10), à une lecture typologique explicitement présentée comme telle. Sa progression et sa place dans l'économie du poème sont remarquables. Conformément au procédé d'annonce observé dans la paraphrase d'Ex 28, les v. 29-30 introduisent les thèmes des §7-8 en évoquant le temps de la Grâce (future gratie signaculum, cf. v. 31) et la divinité du Christ (te deum où l'utilisation de la $2^{\mathrm{e}}$ personne permet de renouer avec l'orientation eucologique

57 Dans l'exégèse traditionnelle d'Ex 28, les Pères, à partir d'Origène, rapportent en effet les quatre étoffes dont sont constitués les vêtements d'Aaron aux quatre éléments dont se compose le monde ; quant aux douze pierres de perfection ornant son rational, elles sont identifiées aux bonnes actions produites par l'âme rationnelle, cf. sur son développement par Grégoire le Grand dans la Règle pastorale 2, 2-4, Judic (1985) 460-463. 
des $\S 1-2$ qui caractérise les $\S 7$ à 10). Puis, la $\S 7$ développe d'un point de vue théorique, en se référant clairement à la méthode typologique (ut, que figure precinebant mistice, / completa in te veritas ostenderet, v. 34-35), l'idée que la figure sacerdotale d'Aaron annonce le Christ, véritable grand prêtre ${ }^{58}$. L'office du Christ, différent de celui des autres grands prêtres, est, dès lors, au centre de la $\S 8$ où le poète reprend les thèmes principaux d'He 7-10 : le Christ, par son sacrifice unique destiné à enlever les péchés de la multitude ${ }^{59}$, a définitivement aboli l'ancien culte et scellé une nouvelle alliance fondée non plus sur la loi, mais sur la grâce. L'affirmation, au v. 40, que l'Église résulte de ce mystère prépare la figure des évêques et des prêtres qu'ils sacrent au centre de la $\S 9$, où ces derniers apparaissent, en tant que guides de l'Église (v. 42), garants de la nouvelle loi (v. 43) et dépositaires de la doctrina (v. 44), à même de sauver leurs ouailles de la mort par la foi en Christ (v. 45). On notera le rôle actif prêté dès la $\S 9$ au Christ dans l'établissement des évêques à la tête de l'Église, avant que la foule de leurs frères ne l'implore, dans la §10, de faire descendre la grâce de l'Esprit sur ses successeurs apostoliques. La boucle est ainsi bouclée avec la § 2 où, dans le prolongement des considérations théologiques de la § 1 sur l'antériorité du Christ au temps par son Père, le Christ était d'emblée présenté, dans son éternité, comme le créateur de tous les sacerdotes depuis Aaron. Parallèlement, l'allusion finale à l'Esprit octroyé par le Christ permet de faire le lien avec le rite de l'ordination, dont l'hymne reprend manifestement, sur un mode lyrique, plusieurs éléments traditionnels.

D'une longue tradition patristique ${ }^{60}$, la typologie sacerdotale des vêtements d'Aaron est en effet au centre des plus anciens rituels d'ordination épiscopale occidentaux. Depuis le sacramentaire Léonien, la prière d'ordination compare l'évêque à Aaron et ses fils aux prêtres, en insistant, comme le suggère l'hymne, sur la transposition qu'a opérée le Nouveau Testament : les rites et les ornements extérieurs, au centre de l'hymne, signifiaient les réalités spirituelles du nouveau sacerdoce (en particulier la pureté des mœurs et des actes) ${ }^{61}$. Même si le texte du rituel épiscopal hispanique est perdu, il est fort vraisemblable que cette typologie y figurait et que le poète anonyme y ait trouvé l'idée d'une paraphrase exégétique d'Exode 28. Les divergences mêmes qu'on observe avec l'interprétation traditionnelle trouvent de toute évidence leur source dans la pratique liturgique. De fait, tout en donnant à

58 Voir, sur cette typologie, Lécuyer (1983) 261-262. On la trouve, à l'époque wisigothique, chez Isidore, De officiis ecclesiasticis II.5.

59 Cf. He 9.15 et 28.

60 Cf. supra n. 57.

61 Cf. ce passage du «Sacramentaire léonien»sur la Consecratio episcoporum (XXVIII) : ... illius namque sacerdotii anterioris habitus nostrae mentis ornatus est et pontificalem gloriam non iam nobis honor commendat vestium sed splendor animorum quia et illa quae tunc carnalibus blandiebantur obtutibus ea potius quae in ipsis erant intellegenda poscebant. [...] hanc quaesumus Dne gratiam largiaris ut quidquid illa velamina in fulgore auri in nitore gemmarum in multimodi operis varietate signabant hoc in horum moribus actibusque clarescat. 
Aaron la place exemplaire qu'il avait dans la tradition liturgique, l'hymne propose une lecture différente de l'interprétation morale traditionnelle faisant, à la lumière de l'Épître aux Hébreux, d'Aaron le modèle non de l'évêque, mais du Christ, véritable sacerdos. Or cette typologie permet, en accord avec un autre motif central des rituels de l'ordination épiscopale, de souligner le rôle essentiel joué par le Christ dans la consécration $^{62}$, tout en lui donnant une place conforme à celle qui est la sienne dans la tradition hymnique, tournée, depuis Hilaire et Ambroise, vers la célébration de son mystère. On notera à cet égard que son invocation se prête, dans la $\S 1$, à des affirmations plus théologiques que dans les hymnes considérés jusque-là : l'image de la «lumière de la lumière» au v. 1 et l'affirmation de l'antériorité du Christ au temps au v. 3 font directement écho au credo de Nicée, dans l'esprit de l'hymne Ante saecula qui manens d'Hilaire dont s'est probablement souvenu l'Anonyme en composant son poème. Mais l'accent théologique que prend à maintes reprises la liturgie hispanique a également dû jouer, d'autant plus que des parallèles précis sont possibles $^{63}$.

Les hymnes de la fin de l'époque wisigothique continuent donc de faire une large place à la doctrine, à des fins désormais purement catéchétiques : les uns traitent, dans la continuité des hymnes d'Ambroise, des grands thèmes christologiques ; les autres empruntent leur inspiration directement à la Bible, alors au centre de la vie religieuse et spirituelle. En dépit de leurs orientations divergentes, tous sont fortement redevables à l'enseignement des Pères, dont ils reprennent non seulement les leçons, mais aussi maintes expressions caractéristiques. Ils s'avèrent ainsi en consonance étroite avec la liturgie de l'époque, qui se signale par son empreinte patristique et théologique. Cette adéquation se vérifie dans le choix des thèmes et des motifs traités, en résonance avec les textes liturgiques du jour, qu'ils reprennent sur le mode de la célébration. Ces hymnes présentent de la sorte un accent nouveau, loin du lyrisme empreint de classicisme d'Ambroise et de la tradition poétique tout court, sinon hymnique. On notera cependant que la part importante de ces sources prosaïques ne nuit guère à leur valeur esthétique, loin s'en faut. La part importante de la doctrine dans ces poèmes, et le choix fait ici d'aborder ces textes sous l'angle doctrinal ne sauraient en effet occulter la charge poétique de ces hymnes, qui facilitait largement l'assimilation de la doctrina. Outre la part importante des images, ces hymnes jouent à maintes reprises des répétitions, des parallélismes et des énumérations, qui concourent à leur musicalité et chargent leur langue simple d'une grâce délicate ; quant à leur écriture rythmée et rimée, elles donnent à leur poésie une intensité nouvelle, plus accessible au peuple, que ces laudes visaient à édifier tout en rendant hommage à Dieu.

62 Cf. Lécuyer (1985) 225-324 («À l'origine de l'ordination : le Christ»).

63 Cf. en particulier les affinités des v. 3-4 avec le passage liturgique cité n. 28. 


\section{Bibliographie}

Arana Tarazona (2016) : Himnos de la liturgia mozárabe. Versión castellana de los himnos de la liturgia mozárabe. Realizada desde la edición de Blume, Clemens, Hymnodia Gotica. Die Mozarabischen Hymnen des alt-spanischen Ritus, obra inédita facilitada a La Ermita en febrero de 2016 (URL : http://www.hispanomozarabe.es/oficio/ofi-himnos.htm\#inicio)

Arocena-Solano (2012): Félix María Arocena-Solano, «El Himnario del Oficio hispano», in Scripta Theologica 44.1, 9-44.

Blume (1897): Clemens Blume (ed.), Hymnodia Gothica. Die Mozarabischen Hymnen des alt-spanischen Ritus, Leipzig (reprint 1961) (AH 27).

Castro Sánchez (2010): José Castro Sánchez (ed.), Hymnodia hispanica, Turnhout (CCSL 167).

Castro Sánchez / García Ruiz (2014): José Castro Sánchez (ed.) / Emilio García Ruiz (trad.), Himnodia hispánica: Introduccion, traduccion, indices y notas, Turnhout (CCT 19).

Charlet (1985): Jean-Louis Charlet, «L'inspiration et la forme bibliques dans la poésie chrétienne $\mathrm{du} \mathrm{II}^{\mathrm{e}}$ au $\mathrm{VI}^{\mathrm{e}}$ siècle», in: Jacques Fontaine / Charles Pietri (dir.), Le monde latin antique et la Bible, Paris, 613-643.

De Almeida Matos (1977): Alibo De Almeida Matos, Hinos do Temporal Hispánico Até à Invasâo Muçulmana: estudo histórico-crítico, Coimbra.

De Almeida Matos (1984): Alibo De Almeida Matos, «Hinario hispânico do Temporal. Hinos mais recentes», in: Revista da Universidade de Aveiro 1, 107-118.

Díaz y Díaz (1965): Manuel C. Díaz y Díaz, «El latin de la liturgia hispanica», in: Juan Francisco Rivera Recio (ed.), Estudios sobre la liturgia mozarabe, Toledo, 55-87.

Díaz y Díaz (1980): Manuel C. Díaz y Díaz, "Literary Aspects of the Visigothic Liturgy», in: Edward James (ed.), Visigothic Spain: New Approaches, Oxford, 61-76.

Díaz y Díaz (1986): Manuel C. Díaz y Díaz, «Noticias históricas en dos himnos litúrgicos visigodos», in: Antigüedad y Cristianismo III. Los visigodos, historia y civilización, Actas [21-25 Oct. 1985], Murcia, 443-456.

Fernández Alonso (1955): Justo Fernández Alonso, La Cura pastoral en la España romanovisigoda, Roma.

Férotin (1912): Marius Férotin, Le Liber mozarabicus sacramentorum et les manuscrits mozarabes, Paris.

Ferro Calvo (1972): Mauricio Ferro Calvo, La celebración de la venida del Señor en el oficio hispánico: estudio histórico doctrinal de los formularios del oficio para el ciclo natalicio en las dos tradiciones del rito hispánico, Madrid.

Fontaine (1974/5): Jacques Fontaine, «L'apport de la tradition poétique romaine à la formation de l'hymnodie latine chrétienne ", in: Revue des Études latines 52, 318-355.

Fontaine (1992): Jacques Fontaine (ed.), Ambroise de Milan, Hymnes, texte établi, traduit et annoté sous la direction de Jacques Fontaine, Paris.

Fontaine (1999): Jacques Fontaine, «Un débat sur les Hymnes en 633 lors du $4^{\text {ème }}$ Concile de Tolède», in: Helmántica 50, 383-402.

Gallego Moya (2002): Elena Gallego Moya (éd.), Los himnos de la Hymnodia Hispanica-Estudio preliminar, traduccion, notas y comentarios, Alicante.

Guéranger (1841): Prosper Guéranger, L'année liturgique. L'Avent liturgique, Mans.

Janeras (1954): Vicente Janeras, «Combinación de los oficios temporal y festivo en la liturgia hispana», in: Archivos leoneses 8, 196-225.

Janini (1980): José Janini (ed.), Liber misticus de Cuaresma y Pascua (Cod. Toledo, Bibl. Capit. 35.5), Toledo. 
Judic (1985): Bruno Judic, «La Bible miroir des pasteurs dans la Règle Pastorale de Grégoire le Grand», in: Jacques Fontaine / Charles Pietri (dir.), Le monde latin antique et la Bible, Paris, 455-478.

Lecuyer (1983): Joseph Lecuyer, Le sacrement de l'ordination, Paris.

Lopetegui Semperena (2005): Guadalupe Lopetegui Semperena, «Poesía latina hispana: lírica religiosa», in: Vitalino Valcárcel Martínez / Carlos Pérez González (eds.), Poesía Medieval (Historia literaria y transmisión de textos), Burgos, 135-180.

Madoz (1938) : José Madoz, Le symbole du Xle Concile de Tolède: ses sources, sa date, sa valeur, Louvain.

Mayer (1927): Anton L. Mayer, «Mater et filia», in: Jahrbuch fur Liturgiewissenschaft, 60-82.

Messenger (1946): Ruth E. Messenger, «Mozarabic Hymns in Relation to Contemporary Culture in Spain», in: Traditio 4, 149-177.

Pinell (1954): Jorge M. Pinell, «Las Missae, grupos de cantos y oraciones», in: Archivos Leoneses 8, 145-185.

Pinell (1955): Jorge M. Pinell, «El Liber Horarum y el Misticus entre los libros de la antigua liturgia hispana», in: Hispania Sacra 8, 85-109.

Pinell (1957): Jorge M. Pinell, «El Oficio hispano-visigotico», in: Hispania Sacra 10, 1-43.

Pinell (1965): Jorge M. Pinell, «Los textos de la antigua liturgia hispánica. Fuentes para su estudio», in: Juan Francisco Rivera Recio (ed.), Estudios sobre la liturgia mozarabe, Toledo, $109-164$.

Ramos Sánchez (1964): Manuel Ramos Sánchez, Oratio admonitionis. Contribución al estudio de la antigua misa española, Granada.

Rovalo (1966): Pedro Rovalo, «Temporal y santoral en el adviento visigodo, su relación a través del Oficio», in: Hispania Sacra 19, 243-321.

Szövérffy (1998): Josef Szövérffy, Iberian Hymnody. Survey and problems, Leiden.

Urlacher-Becht (2012): Céline Urlacher-Becht, «Les recueils hymniques du poète latin Prudence. Des livres poétiques destinés à être goûtés par un lecteur aux chants liturgiques», in: Greta Komur-Thilloy / Anne Réach-Ngô (ed.), L'écrit à l'épreuve des médias du Moyen Âge à l'ère électronique, 75-91.

Urlacher-Becht (2014): Céline Urlacher-Becht, Ennode de Pavie, chantre officiel de l'Église de Milan, Paris.

Vives (1946): José Vives (ed.), Oracional visigótico. Edición crítica por José Vives ; estudio paleográfico de los códices por Jerónimo Claveras, Barcelona. 
